\title{
A NOTE ON COMPLETIONS OF MODULES
}

\author{
JOSEPH J. ROTMAN
}

Let $R$ be a discrete valuation ring, i.e., a local principal ideal domain. In what follows, module shall mean unitary $R$-module. Following Kulikov, any module $M$ contains a basic submodule $B,{ }^{1}$ i.e., a pure submodule which is the direct sum of cyclic modules and such that $M / B$ is divisible. Basic submodules need not be unique, but any two basic submodules of a module $M$ are isomorphic. We now assume that $M$ has no elements of infinite height, i.e., $\cap p^{n} M=0$. Under this assumption, the submodules $p^{n} M$ can be considered as a system of neighborhoods of 0 making $M$ into a metric topological module. Thus we may form the completion $M^{*}$ of $M ; M$ is complete if $M^{*}=M$. If a submodule $S$ of $M$ is pure, then the topology on $S$ induced by the system of neighborhoods $p^{n} S$ is the same as that induced on $S$ considered as a subspace of $M$. Also a submodule $S$ is dense in $M$ iff $M / S$ is divisible. In particular, a basic submodule is a dense subspace.

Along with some new theorems, we also include some new short proofs of old theorems in the above spirit.

TheOREM 1. Any complete module $M$ is the completion of a direct sum of cyclic modules. Two complete modules are isomorphic if they have isomorphic basic submodules.

Proof. Let $M$ be complete with basic submodule $B$. Since $B$ is a subspace, $B^{*} \subset M^{*}=M$. But $B$ is dense, and so $B^{*}=M$. The second statement follows from the fact that any two basic submodules of $M$ are isomorphic.

Theorem 2. If $S$ is a pure complete submodule of $M$, then $S$ is a direct summand of $M$.

Proof. Let $B$ be a basic submodule of $S$; since $S$ is pure, we may extend $B$ to a basic submodule $C$ of $M$, and $B \oplus B^{\prime}=C$. Since all topologies match, by purity, $S=S^{*}=B^{*}$, since $S$ is complete. But $M^{*}=C^{*}=\left(B \oplus B^{\prime}\right)^{*}=B^{*} \oplus B^{*}=S \oplus B^{\prime *}$. Thus $S$ is a summand of $M^{*}$, a fortiori, a summand of $M$.

TheOREM 3. Any reduced torsion-free $R^{*}$-module $M$ of finite rank is free.

Received by the editors August 28, 1959.

1 The standard existence theorem is for modules over complete discrete valuation rings, but it is easy to see the completeness is unnecessary. 
Proof. Induction on the rank $r$ of $M$. If $S$ is pure of rank $r-1$, then $S$ is free, by induction. Since $R^{*}$ is complete and the rank of $S$ is finite, $S$ is complete. By Theorem 2, $S$ is a summand of $M$. Hence $M$ is free.

In investigating structure problems of torsion-free $R$-modules, I came across the following phenomenon. Let $M$ be an indecomposable torsion-free $R$-module of rank 2 of the type exhibited by Kaplansky in [2]. If we form the completion we find $M^{*} \cong R^{*}$; the rank decreases. Incidentally, this example shows that there is no connection between taking the completion of a module and tensoring the module by the completion of the ring, $R^{*}$. Basic submodules explain this lowering of rank.

Theorem 4. Let $M$ be a torsion-free $R$-module of finite rank with basic submodule B. Then $\operatorname{rank}_{R} M^{*}=\operatorname{rank}_{R}$ B. Further, $\operatorname{rank}_{R^{*}} M^{*}$ $=$ rank $_{R} M$ iff $M$ is free.

Proof. Since $B$ is dense in $M, M^{*}=B^{*}$. Since $B$ is free of finite rank, $B^{*}=(\Sigma R)^{*}=\Sigma R^{*}$, so that $\operatorname{rank}_{R^{*}} M^{*}=\operatorname{rank}_{R} B$. If $\operatorname{rank}_{R^{*}} M^{*}$ $=\operatorname{rank}_{R} M$, then $\operatorname{rank}_{R} M=\operatorname{rank}_{R} B$. However, a pure submodule $B$ of $M$ of the same rank must be $M$, i.e., $B=M$, and so $M$ is free.

We now show the algebraic structure of a complete module.

Lemma 5. Let $B=\Sigma C_{\alpha}, C_{\alpha}$ cyclic. Then $B^{*}$ is the submodule of $\Pi C_{\alpha}^{*}$ consisting of all elements $x=\left\{x_{\alpha}\right\}$ whose coordinates are countably nonzero, and, given any subset of the coordinates, almost all are divisible by $p^{n}$, for any integer $n$.

Proof. It is straightforward to check that the above submodule is complete and contains $B$ as a pure dense submodule.

Corollary. Let $B=\Sigma C_{\alpha}$. $B^{*}$ is a summand of $\Pi C_{\alpha}^{*}$.

Proof. By Theorem 2, we need only show that $B^{*}$ is pure in $\Pi C_{\alpha}^{*}$. But this is obvious, by Lemma 5 .

COROLlaRy. $\Sigma R^{*}$ is contained in a proper summand of $\Pi R^{*}$.

The above corollary is false for abelian groups. Los has shown that the weak direct sum of countably many copies of the integers is contained in no proper summand of the strong (complete) direct sum of the integers.

Lemma 6. Let $M$ be a complete module with submodule $S . M / S$ has no elements of infinite height iff $S$ is closed.

Proof. $\Rightarrow$ Let $\pi: M \rightarrow M / S$ be the natural homomorphism. Since 
$M / S$ has no elements of infinite height, 0 is closed in the $p$-adic topology in $M / S$, and so $S=\pi^{-1}(0)$ is closed in $M$.

$\Leftarrow$ Suppose $p^{n} b_{n}^{*}=a^{*}$, for all $n$, where ${ }^{*}$ denotes coset $\bmod S$. Then $p^{n} b_{n}+s_{n}=a, s_{n} \in S$. Hence $p^{n+1} b_{n+1}+s_{n+1}-\left(p^{n} b_{n}+s_{n}\right)=0$, and so $s_{n+1}-s_{n} \in p^{n} M$. Hence $\left\{s_{n}\right\}$ is Cauchy and so there is an $x \in M$ such that $s_{n} \rightarrow x$, since $M$ is complete. Since $S$ is closed, $x \in S$. Hence $p^{n} b_{n}+s_{n}-x=a-x$. Taking the limit, the left side tends to 0 . Hence $a=x$, i.e., $a \in S$. Therefore $a^{*}=0$.

Theorem 7. Let $M$ be complete, $S$ a closed submodule. Then $M / S$ is complete.

Proof. By the lemma, $M / S$ is a metric space. Suppose $\left\{a_{n}^{*}\right\}$ is Cauchy in $M / S$, i.e., $a_{n+1}^{*}-a_{n}^{*}=p^{k(n)} b_{n}^{*}$, and $k(n) \rightarrow \infty$. By induction on $n$, we show that there are representatives $a_{n}$ of $a_{n}^{*}$ such that $a_{n+1}-a_{n} \in p^{k(n)} M$. Select $a_{1}$ arbitrarily so that $a_{1} \rightarrow a_{1}^{*}$. Suppose we have chosen $a_{i}, i \leqq n$, such that $a_{i}-a_{i-1} \in p^{k(i-1)} M$. Lifting $a_{n+1}^{*}$ arbitrarily to $a_{n+1}^{\prime}$, we obtain $a_{n+1}^{\prime}-a_{n}=p^{k(n)} b_{n}+s_{n}, s_{n} \in S$. Define $a_{n+1}=a_{n+1}^{\prime}-s_{n}$. Then $a_{n+1} \rightarrow a_{n+1}^{*}$, and $a_{n+1}-a_{n} \in p^{k(n)} M$. Since at each step we are extending the given set of representatives, we have constructed a sequence $\left\{a_{n}\right\}$ in $M$ such that $a_{n} \rightarrow a_{n}^{*}$ and $\left\{a_{n}\right\}$ is Cauchy in $M$. Since $M$ is complete, $\lim a_{n}=a$. Since $\pi: M \rightarrow M / S$ is continuous, $\lim a_{n}^{*}=a^{*}$. Hence $M / S$ is complete.

As an application of Theorem 7, we now give a conceptually simple proof of a theorem of Baer, Hilfssatz 4.1, [1]. The question under consideration was: does the group of sequences of integers $\Pi$, have the property that if $G / T \cong \Pi$, then $T$ is a direct summand, where $T$ is the torsion subgroup of $G$ ? In attacking this problem, Baer needed the following crucial lemma. For fixed prime $p$, let $C$ denote the subgroup of $\Pi$ consisting of all elements almost all of whose coordinates are divisible by $p^{n}, n$ any integer $>0$. If $T$ is $p$-primary with no elements of infinite height, and $f: C \rightarrow T$, then $f(C)$ has bounded order. We now prove this lemma.

Proof. Let $G$ be an abelian group with no elements of infinite $p$ height, $p$ a fixed prime. We equip $G$ with the $p$-adic topology and form its completion $G^{*}$. If $G$ is the integers, $G^{*}$ is the $p$-adic integers. If $G$ is free of countable rank, then $G^{*}$ consists of all sequences in a strong direct sum of a countable family of copies of the $p$-adic integers such that almost all of its terms are divisible by $p^{n}, n$ any integer $>0$. Thus $C=\Pi \cap(\Sigma \text { integers })^{*}$. Note that both the completion and $T$ are modules over the $p$-adic integers.

We claim that $f: C \rightarrow T$ can be extended to $\Sigma^{*}$, the completion of the free group. If $y \in \Sigma^{*}$, then $y=\left\{y_{i}\right\}$. If $y$ has only one nonzero 
coordinate $y_{i}$, we define $f(y)$ as follows: suppose $p^{k} f\left(x_{i}\right)=0$, where $x_{i}$ is a generator of the $i$ th factor of $\Pi$. Now $y_{i}=\delta x_{i}, \delta$ a $p$-adic integer. Take integers $r_{n}$ such that $r_{n} \rightarrow \delta$. For large $n, \delta-r_{n} \in\left(p^{k}\right)$. Define $f(y)=f\left(r_{n} x_{i}\right)$. Now consider a general $y$. For each $i$, choose an integer $r_{i}$ such that $f\left(y_{i}\right)=f\left(r_{i} x_{i}\right)$. Define $f(y)=f\left(\left\{r_{i} x_{i}\right\}\right)$. It remains to prove independence of choices. Suppose $f\left(r_{i} x_{i}\right)=f\left(r_{i}^{\prime} x_{i}\right)$ for all $i$. Claim $f\left(\left\{\left(r_{i}-r_{i}^{\prime}\right) x_{i}\right\}\right)=0$. If $y \in C, h\left(y-\sum_{i=1}^{k} y_{i}\right) \rightarrow \infty$, where $h$ is the height function. Hence $h\left(f(y)-\sum_{i=1}^{k} f\left(y_{i}\right)\right) \rightarrow \infty$. If $f\left(y_{i}\right)=0$ for all $i$, then $h(f(y))$ is infinite. Since $T$ has no elements of infinite height, $f(y)=0$. Thus the extension is well-defined. It is easy to check that $f$ is a $p$-adic integer homomorphism.

By Theorem $7, f\left(\Sigma^{*}\right)$ is complete. But the only complete torsion modules have bounded order. In particular, $f(C)$ is of bounded order.

Lemma 8. Let $M$ be a complete module with basic submodule $B$, and let $T$ be a module with no elements of infinite height. If $f: B \rightarrow T, f$ is extendable to $M$ iff $(f(B)) * C T$.

Proof. $\Rightarrow$ If $f$ is extendable, $f(M)$ is complete. Hence $(f(B))^{*}$ $C(f(M))^{*}=f(M) \subset T$.

$\Leftarrow$ Suppose $(f(B))^{*} \subset T$. Let $\left[x_{\alpha}\right]$ be an independent generating set of $B$. If $y \in M, y=\left\{r_{\alpha} x_{\alpha}\right\}, r_{\alpha} \in R$. Further, there is a sequence $\left\{\alpha_{i}\right\}$ of distinct indices such that $r_{\alpha}=0$ if $\alpha \neq \alpha_{i}$ for some $i$, and also almost all the $r_{\alpha_{i}}$ are divisible by $p^{n}$ for any positive integer $n$. Hence $f\left(r_{\alpha_{i}} x_{\alpha_{i}}\right) \rightarrow 0$ in $f(B)$. Thus $\left\{\sum_{i=1}^{k} f\left(r_{\alpha_{i}} x_{\alpha_{i}}\right)\right\}$ is Cauchy in $f(B)$. Therefore there is a $z \in(f(B))^{*} \subset T$ such that $\sum_{i=1}^{\infty} f\left(r_{\alpha_{i}} x_{\alpha_{i}}\right)=z$. Define $f(y)=z$.

Theorem 9. Let $M$ be complete with basic submodule $B, T$ a complete module. Then any $f: B \rightarrow T$ has a unique extension over $M$.

Proof. An extension exists, by the lemma. It is unique since any homomorphism is continuous, and $B$ is dense.

Corollary. Let $M$ be complete with basic submodule $B, T$ a complete module. Then $\operatorname{Hom}(M, T) \cong \operatorname{Hom}(B, T)$, the isomorphism being induced by the inclusion map $j: B \rightarrow M$.

Given $f: B \rightarrow T$ (notation the same as in Theorem 9), one may ask whether the extension $g: M \rightarrow T$ inherits any of the properties of $f$, or vice versa.

Lemma 10. If $B$ is basic in $M, M$ and $T$ complete with $f: M \rightarrow T$, then $f\left(B^{*}\right)=(f(B))^{*}$.

Proof. $(f(B))^{*} C(f(M))^{*}=f(M)=f\left(B^{*}\right)$. On the other hand, if 
$s \in f\left(B^{*}\right)$, then there is a $b \in B^{*}$ such that $f(b)=s$, and there is a sequence $\left\{b_{n}\right\}$ in $B$ such that $b_{n} \rightarrow b$. Hence $f\left(b_{n}\right) \rightarrow f(b)=s$. Hence $s \in(f(B))^{*}$.

THEOREM 11. With the notation above, if $f$ is an epimorphism, so is $g$; if $g$ is a monomorphism, so is $f$.

Proof. Trivial, using Lemma 10.

More interesting is the fact that both converses of Theorem 11 are false.

Let $B$ be free of countable rank, and let $B^{\prime} C B$ be such that $B / B^{\prime}$ is the quotient field of $R$. Thus $B^{\prime}$ is basic in $B$. Let $M=B^{*}$. Both $B$ and $B^{\prime}$ are basic submodules of $M$. Let $f: B \rightarrow B$ be a monomorphism with $\operatorname{im} f=B^{\prime}$, and let $g: M \rightarrow M$ extend $f . g$ is an epimorphism, since im $g$ is complete and contains the dense submodule $B^{\prime}$. However $f$ is not an epimorphism.

Let $B$ be free on generators $x_{i}, i=1,2, \cdots$, and let $M=B^{*}$. Define $f: B \rightarrow B$ by $f\left(x_{1}\right)=p x_{1} ; f\left(x_{k+1}\right)=p x_{k+1}-x_{k} . f$ is a monomorphism, and it is easily checked by induction that $f\left(p^{k} x_{k}\right)=p^{k+1} x_{k}$ $-\sum_{i=1}^{k-1} f\left(p^{i} x_{i}\right)$. Now $y=\left\{p^{i} x_{i}\right\} \neq 0 \in M$. Further $\Sigma p^{i} x_{i} \rightarrow y$, and so $f\left(\Sigma p^{i} x_{i}\right) \rightarrow g(y)$. But $\Sigma^{k} f\left(p^{i} x_{i}\right)=f\left(p^{k} x_{k}\right)+\Sigma^{k-1} f\left(p^{i} x_{i}\right)=p^{k+1} x_{k} \rightarrow 0$. Hence $g(y)=0$, and $g$ is not a monomorphism.

\section{REFERENCES}

1. R. Baer, Die Torsionsuntergruppe einer abelschen Gruppe, Math. Ann. vol. 135 (1958) pp. 219-234.

2. I. Kaplansky, Infinite Abelian groups, Ann Arbor, University of Michigan, 1954.

University of Chicago 